\title{
Research on Design and Development of Mobile Serious Game under Mobile Learning Environment
}

\author{
Husheng Pan $^{1}$, Chenjie Guo ${ }^{2}$, Junfu Yu ${ }^{2}$, Yuehua Chen ${ }^{1, *}$ \\ ${ }^{1}$ School of Mechatronic Engineering, Harbin Institute of Technology, Harbin, 150000, China \\ ${ }^{2}$ School of Mechanical Engineering, Shandong University, Jinan, 250000, China \\ *Corresponding Author: Yuehua Chen
}

Keywords: Mobile serious game, Mobile learning environment

\begin{abstract}
Mobile learning based on cellphone has become more and more popular among modern learners. Serious games not only create rich learning environments for learners, but also stimulate learners' interest in learning. This paper presents the design strategies of mobile serious games, including character design strategies, scene design strategies and interaction design strategies. Taking the game oriented cultivation of children's cooperation ability as an example, the paper explores the key points of the development and test of mobile serious games under mobile learning environment to provide some references for the relative researchers.
\end{abstract}

\section{Introduction}

With the rapid development of mobile communication technology and wireless internet technology, a new learning mode named mobile learning is quietly emerging. The characteristics of personalized learning at anytime and anywhere attract the attention of many scholars at home and abroad. However, it has become an urgent problem to ensure learners to focus on learning in a mobile learning environment for a long time, and that fragmentation learning can be effectively integrated into system learning. It is a meaningful attempt to integrate edutainment into mobile learning.

At the same time, with the implementation of $4 \mathrm{G}$ technology, mobile games have made great leaps both in the game quality and the game platform. It also provides great opportunities for the combination of serious games and mobile learning.

Serious games are games that are not just for entertainment purposes. After the integration of the viewpoints of many experts, the author believes that serious game is not design for entertainment, but designed to achieve the new digital education, training, health care and other purposes through the look and feel of a game true simulation of the real event or process. Serious game is a new research field that mass media is born with the new media now. It is a new generation of digital games, but also in the media after the broadcast drama, opera, television and animation and other forms of entertainment after a new form of media communication. In recent years, it has been widely used in Europe, North America and other developed countries, and has a big or small impact on people's daily life. However, the idea of the serious game was introduced into China only several years ago, and the propaganda of the enterprise market is lagging. Therefore, the recognition of serious games in our country is not particularly optimistic.

Mobile serious game is serious game in the mobile phone on the realization of the carrier. It has the characteristics of mobile games and serious games, and has unique advantages in portability and entertainment. Users can use mobile phones to play games at any time and place, actively acquiring knowledge and skills, and achieving changes in attitudes and behaviors.

\section{Design Strategies of Mobile Serious Games under Mobile Learning Environment}

Character Design Strategies. The designer's design process is to seek out the original image of the characters from some of the salient features of the characters, and to enrich the general characteristics of the characters based on the characteristics, requirements, and performance of the 
general tasks in the game. This is a process of the full role of the entire game process. The game player is choosing the characters to play. The physiological characteristics, the role of the social background and other attributes are presented in front of game player. A game player will enjoy the role of the potential function in-depth along with the growth of the role. In the design of the game, the role image is the first step and the most important step of the game for the player to play. In designing the game characters, the following aspects can be considered. First, the role image must be able to arouse the interest of the player. For the player, the intuitive perception of the characters of the game can greatly influence whether the player plays the game. Second, the characters must be liked by the players. It is the initial emotional experience of the serious game for the player. If the player does not cultivate any feelings of the character in the process of the game, he won't have any attachment to the game. There is no desire to continue the serious game. Once again, the characters can grow or change as the game goes on, so that the game characters are more realistic, and the players can choose the characters' appearance, shape, skills and so on according to the growth of the characters. The constant growth of the characters in the serious game has a profound experience for the emotions of the players, which is very conductive to the learning of the players.

Scene Design Strategies. Learning is conducted in a certain situation, and it is also important to design a game scene that is conducive to the emergence and development of knowledge. Game scenes illustrate the history of the game, the time, the natural environment, the characters, the places of life, and so on, for the game to create a mood and atmosphere. The scene design in the serious game can be considered from two aspects: the scene atmosphere and the scene arrangement. The atmosphere of the scene refers to the tone of the game, such as romantic, realistic, historical, cartoon and so on. When setting the atmosphere for the cartoon scene, we usually use cute, exaggerated, bright color scene. If it is a historical theme of the serious game, it is not suitable for the bright color. We should adopt the steady cold tone series of color to create a serious atmosphere. The layout of the scene refers to the placement of the objects in the serious game scene. On the other hand, the objects in the scene are also able to represent the themes of the game. The stimulation and the maintenance of the motivation are the important factors in inquiry game learning activity. Influenced by the serious game situation and the serious game task, the player will have a new understanding of the role, and under the common cultural characteristics. The role bears the task of conveying the intention of empathy. A feedback scene in Finding Nemo is shown as follows. When a small fish was taken by divers, a close-up set of fish dads impressed many audiences. Scene navigation is more common in mobile serious games. It differs from the traditional navigation in the layout of the interface, showing the arena entrance of the big icons. The information is easy to convey while allowing players to race quickly into battle. The navigation model of the scene design can greatly enhance the immersion feelings of the serious game.

Interaction Design Strategies. In the serious games activities, the cognitive process occurs in the process of the interaction between the player and the game content and interaction skills. The students continue to use has been mastered knowledge to complete the task in the playing process. It not only achieves the exploration of new knowledge, but also has a test of the original knowledge in the process of completion of the game. But the emotional interaction between students and teachers is changed into the player in the game to get emotional support, evaluation of the students during the game, let them get some psychological suggestion, this suggestion for encouragement, praise, affirmations, through the emotional interaction, optimize learning effect. It should be noted that in the evaluation, we should not only pay attention to the evaluation of intelligence factors, but also pay attention to the evaluation of nonintellectual factors. In the evaluation, affirmative suggestion often makes the student accept easily, can promote the student to study further, making progress toward the higher goal. Conversely, if more negative and negative words are used to stimulate the player, it can produce undesirable side effects. Creating a happy and harmonious learning atmosphere helps to keep the player learning. The amount of interaction refers to the number of times per unit time during the game. The quality of interaction is that during the game, these interactions are meaningful, positive and effective for learning. With the development of internet and computer, the frequency of interaction in the game increases obviously, which provides more feedback for the learners' learning, and helps to keep learners' interest in learning. However, studies show that it is not a fact that more 
interaction, higher teaching quality. There is not necessarily proportional relationship between the two factors. Too many easy interactions may cause the students too busy and too few interactions may lead to the isolated feelings of the learners. Therefore, the best way is to provide a considerable amount and quality of learning interactions according to the requirements of the learning objectives in the serious games.

\section{Development and Test of Mobile Serious Games under Mobile Learning Environment}

Taking the game oriented cultivation of children's cooperation ability as an example, the paper explores the key points of the development and test of mobile serious games under mobile learning environment.

Platform Construction. The founder of development environment of Android is the geek of Silicon Valley named Andy Rubin. The Android Development Tools are the development tools under the IDE environment based on Android to provide the upgrade of the development tools of Android. We can install the ADT plug-in unit via the network site of developer.android.com in the form of online installation. The process is shown as follows.

1. Start the Eclipse and choose the menus of Help > Install New Software

2. Click the Add button in the top right-hand corner.

3. Input "ADT Plugin" in the location of Name and the site of https://dl-ssl.google.com/android/eclipse/.

4. Click OK.

5. Choose "Developer Tools" in the dialog box, and then click "Next".

6. In the next window, you will see a tool list. Click "Next".

7. Read and accept the agreement and then click "Finish"

8. Reset Eclipse after installation.

Achievement of Mobile Serious Game. Children click the game system icon button oriented cultivation of children's cooperation ability to enter the game landing interface. The login interface is natural and fresh, with natural scenery as the background, which is close to children, love and nature. At the same time, the registration interface of educational game system for children's cooperative ability conforms to the characteristics of children's cognition and the design principles of color, shape and pattern elements of children's cooperative educational games.

When children register and sign up for an educational game system for children's cooperative ability, they can inquire about the basic situation. The information includes names, gender, age, class and so on. The information shows that the original login interface as the background. The content appears translucent above the background, which can give children to feel naturally elegant, switch interface. It will not give children a sense of children caused by abrupt visual differences huge complete cooperative education after the game. They can enquiries cooperative game performance information. The information includes the cooperative game name, the game time, the difficulty and so on. The form of the interface form is simple and natural, and can give children and parents direct feedback.

The jigsaw puzzle is to break up the entire picture or model and to restore to the original picture or model pieces in accordance with certain ways together. We introduce the methods and rules of this ancient game into the field of teaching. The operation of the game mainly includes the child's click and drag operation. This is one of the most common habits of children, consistent with the characteristics of children's cooperative education games. During the game, when children make correct or incorrect operations, the system can give prompt speech or animation hints. It conforms to the key elements of children's cooperative education game sound and reward strategies. After children's cooperative games, they can inquire about the scores of their cooperative games, and provide a good way for children and parents to give feedback. It also fully fits the interactive feedback principle of children's human-computer interaction design.

From the comprehensive view, the system is designed with the children as the center. In the process of cooperative game design, the design will integrate elements of children's natural human-computer interaction design principles and children's cooperative education game to create a 
real cooperative game situation. It allows children to immerse themselves in the fun of the game, and ultimately realize the children's cognition, understanding, imagination, communication, cooperation and other all-round training of multiple intelligences.

Test of Mobile Serious Game. After designing and implementing an educational game system for children's cooperative abilities, we used the system to test 15 groups of 3-6-year-old children. The results are shown as follows.

Table 1. Initial finish time and Finishing time after training of different children groups

\begin{tabular}{|c|c|c|}
\hline Number & Initial finish time (s) & Finishing time after training (s) \\
\hline 1 & 84 & 67 \\
\hline 2 & 75 & 62 \\
\hline 3 & 72 & 69 \\
\hline 4 & 81 & 76 \\
\hline 5 & 71 & 72 \\
\hline 6 & 72 & 77 \\
\hline 7 & 79 & 60 \\
\hline 8 & 73 & 61 \\
\hline 9 & 71 & 74 \\
\hline 10 & 72 & 70 \\
\hline 11 & 80 & 68 \\
\hline 12 & 77 & 69 \\
\hline 13 & 81 & 60 \\
\hline 14 & 83 & 83 \\
\hline 15 & 74 & 64 \\
\hline
\end{tabular}

According to the Table 1, we draw the Line chart as follows:

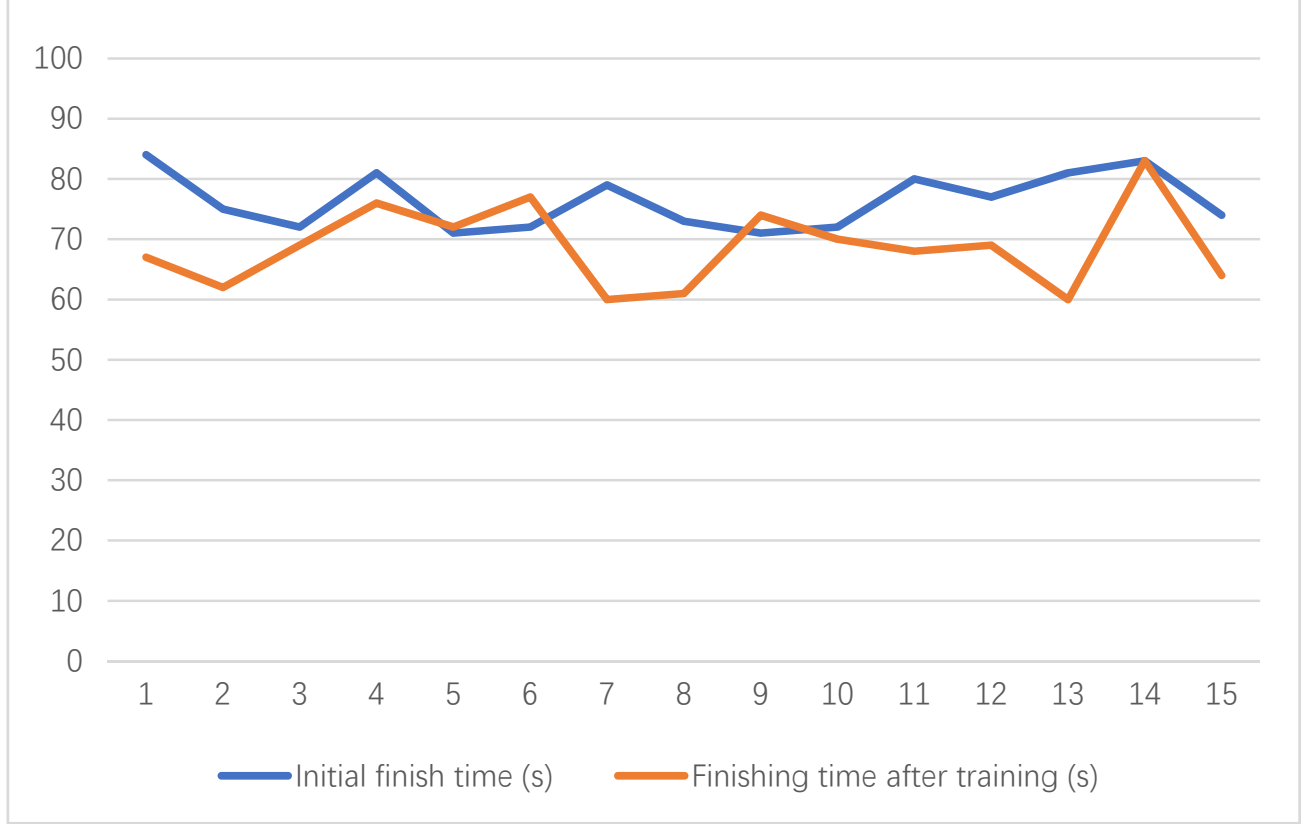

Figure 1. Initial finish time and finishing time after training of different children groups

This result shows that children have better performance of the cooperation level and ability to some extent after using the educational game system which is oriented cultivation of children's cooperation ability. 


\section{Conclusion}

Considering the differences between mobile phones and computers, this paper puts forward some design principles for mobile educational games, and pays attention to them in the process of implementation. Today, the research on the mobile phone serious games has just started. As the mobile phone functions becomes more powerful, and the serious game technology has become more sophisticated, we believe that mobile phone serious games will be applied more widely. And its value will be further reflected.

\section{Acknowledgements}

This paper is the stage results of the National Social Science Fund Project named Serious Game Spread Socialist Core Values of the Design Strategy and Mode of Operation (Grant No. 16BXW004).

\section{References}

[1] ZhouWenjie, Mu Rui. A Study on the Effects of Serious Games on Cross-Cultural Learning and Behavior Coordination [J]. Journal of Shenyang Normal University (Social Science Edition), 2016(6): 137-141.

[2] ZhouWenjie, Mu Rui. Case-based Study on the Role of Serious Games in Cultivating Undergraduates Cooperative Consciousness [J]. Journal of Northeastern University (Social Science), 2017, 19(2): 201-207.

[3] Chang Jun, Wang Fan. Opportunities and Challenges of Phone Serious Games in Mobile Learning Environment [J]. China Educational Technology \& Equipment, 2013(6): 14-15.

[4] Wang Xuan. Mobile Terminal Serious Games Interface Design Studies for Children [J]. Art \& Design, 2014(2): 110-111. 